\title{
Visualization of phage DNA degradation by a type I CRISPR-Cas system at the single-cell level
}

\author{
Jingwen Guan ${ }^{1,2,3}, \mathrm{Xu} \mathrm{Shi}^{1,2}$, Roberto Burgos ${ }^{1}$ and Lanying Zeng ${ }^{1,2,3, *}$ \\ 1 Department of Biochemistry and Biophysics, Texas A\&M University, College Station, TX 77843, USA \\ 2 Center for Phage Technology, Texas A\&M University, College Station, TX 77843, USA \\ 3 Molecular and Environmental Plant Sciences, Texas A\&M University, College Station, TX 77843, USA \\ * Correspondence: Izeng@tamu.edu
}

Received September 20, 2016; Revised December 22, 2016; Accepted February 4, 2017

\begin{abstract}
Background: The CRISPR-Cas system is a widespread prokaryotic defense system which targets and cleaves invasive nucleic acids, such as plasmids or viruses. So far, a great number of studies have focused on the components and mechanisms of this system, however, a direct visualization of CRISPR-Cas degrading invading DNA in real-time has not yet been studied at the single-cell level.

Methods: In this study, we fluorescently label phage lambda DNA in vivo, and track the labeled DNA over time to characterize DNA degradation at the single-cell level.

Results: At the bulk level, the lysogenization frequency of cells harboring CRISPR plasmids decreases significantly compared to cells with a non-CRISPR control. At the single-cell level, host cells with CRISPR activity are unperturbed by phage infection, maintaining normal growth like uninfected cells, where the efficiency of our antilambda CRISPR system is around $\mathbf{2 6 \%}$. During the course of time-lapse movies, the average fluorescence of invasive phage DNA in cells with CRISPR activity, decays more rapidly compared to cells without, and phage DNA is fully degraded by around 44 minutes on average. Moreover, the degradation appears to be independent of cell size or the phage DNA ejection site suggesting that Cas proteins are dispersed in sufficient quantities throughout the cell.

Conclusions: With the CRISPR-Cas visualization system we developed, we are able to examine and characterize how a CRISPR system degrades invading phage DNA at the single-cell level. This work provides direct evidence and improves the current understanding on how CRISPR breaks down invading DNA.
\end{abstract}

Keywords: bacteriophage lambda; CRISPR-Cas; fluorescence microscopy; single-cell analysis; type I CRISPR

\section{INTRODUCTION}

CRISPR-Cas system is a widespread adaptive immune system in prokaryotes including almost half of bacteria and most archaea [1]. Short fragments of foreign DNA, or spacers, are integrated into Clustered, Regularly Interspaced Short Palindromic Repeat (CRISPR) loci on the host genome. The prokaryotes then apply the acquired spacers, which serve as memory elements, to target and

This article is dedicated to the Special Collection of Synthetic Biology, Aiming for Quantitative Control of Cellular Systems (Eds. Cheemeng Tan and Haiyan Liu). cleave invading nucleic acids to defend against viruses and plasmids [1-5]. In the CRISPR loci, the spacers are separated by host-derived, short, repeated sequences, or repeats. Other important components of CRISPR system include CRISPR-associated (cas) genes and a leader sequence, which are found near the CRISPR region. There are multiple kinds of cas genes associated with a specific CRISPR [2,6]. During CRISPR action, the repeat-spacer array is first transcribed into a single transcript, which is further processed by Cas proteins into small RNAs (CRISPR RNA, or crRNA) $[4,7,8]$. The mature crRNA and Cas proteins then assemble to form multicomponent CRISPR ribonucleoprotein (crRNP) 
complexes. The crRNP scans invading DNA by base pairing and on successful recognition, and then the target is eventually degraded by Cas nucleases $[6,9]$.

According to the recent classification of CRISPR-Cas systems, there are two classes with fundamental differences in the organization of the effector module [10]. Class 1 systems include types I, III and IV, and class 2 systems include types II, V, and VI [1,5]. Much of our understanding of type I system is gained from the studies of type I-E system of E. coli K12 [6,11]. Recently, research has significantly advanced our knowledge about the less understood processes such as the acquisition of new spacers, for example, how the new spacers are produced and integrated into the CRISPR arrays [5,1214]. In addition, the mysteries of the structures and mechanisms of the target recognition have been largely uncovered recently [3,15-19]. In the E. coli type I-E system, there is a surveillance complex known as Cascade (CRISPR-associated complex for antiviral defense), a 405-kD complex of short crRNAs and five Cas proteins [20]. Cascade binds to target DNA sequences and recruits Cas3 enzyme (a trans-acting nuclease-helicase, the signature Cas protein for type I system) to unwind and degrade the bound foreign DNA [11,16,19]. Recently, in vitro studies showed that linear DNA target is degraded in a unidirectional manner, and the degradation rate of negatively supercoiled DNA is 4.5 fold faster than the linear one [11]. However, direct demonstration of DNA cleavage in vivo has not been provided yet.

Here, we performed in vivo experiments with the type I-E CRISPR system of E. coli at the single-cell level. An artificial, plasmid-based CRISPR system was introduced into $E$. coli to target bacteriophage lambda [20], where we monitored CRISPR action by infecting cells with our phage strain. With our reporter system, we were able to track in vivo lambda DNA degradation over time under the fluorescence microscope. This work provides insights on how CRISPR breaks down invading DNA.

\section{RESULTS}

\section{Phage lysogenization is significantly reduced with the CRISPR system}

For phage lambda, it was found that the $E$. coli CRISPR system protects against lambda lysogenization, where the lysogenization frequency is 100 -fold lower in the presence of the CRISPR compared to without [21]. To test the efficiency of the artificial CRISPR system in our E. coli phage DNA reporter host cell BA16 (or MG1655 seqA-yfp dam ${ }^{-}$[22], we utilized the same CRISPR plasmids: pWUR397A (cas3) [21], pWUR400 (casABCDE) and pWUR478 ( $\lambda$ phage targeting spacers) /pWUR477 (control spacers) [20]. The CRISPR plasmid
pWUR478 contains four anti-lambda spacers with homology to lambda genes $J, O, R$, and $E$. The control plasmid pWUR477 contains spacers without homology to lambda genome. To examine the function of this CRISPR system against the phage lysogenization, we performed experiments with the CRISPR or control plasmids at two different APIs (average phage input, i.e., average number of phages per cell) through a bulk lysogenization assay [23]. Following the function of the CRISPR-Cas system, foreign phage DNA should be degraded and be unable to integrate into the host cell genome, resulting in reduced efficiency of lysogenization. As shown in Figure 1A, the lysogenization frequency of our host cells with the CRISPR or control plasmids is very low, within the order of $10^{-7}-10^{-3}$ (the colony forming units, or cfu, and plaque forming units, or pfu, counts together with API and the calculated lysogenization frequencies are listed in Supplementary Table S1). To determine the efficiency of this CRISPR-Cas system, we normalized the lysogenization frequency to that of the cells containing the control plasmid within two categories, i.e., slow API (1-6) and high API (15-65). The lysogenization of the CRISPR targeting plasmid is only about $2 \%-7 \%$ as efficient compared to the control, as shown in Figure 1B. This reduced lysogenization frequency indicates that the artificial CRISPR-Cas system works at the bulk level. However, the 2\%-7\% efficiency in lysogenization is slightly higher than the $1 \%$ of previously reported $h n s$ mutant strain harboring the same CRISPR plasmids at API of 1 [21]. This could be due to our host strain MG1655 containing the $h n s$ product, heat-stable nucleoid structuring (H-NS) protein, a global transcriptional repressor in E. coli, or other host differences in our phage DNA reporter strain, for example, our cells with the $\mathrm{dam}^{-}$allele lack host DNA methylation, thereby abrogating the regulation of $E$. coli replication initiation by SeqA [24-27].

\section{Visualizing CRISPR function in single cells}

To visualize the phage DNA degradation process, we employed a reporter system for visualizing infecting phage particles on the cell surface, and phage DNA inside the cell [23]. The infecting phage $\lambda_{\mathrm{LZ760}}$ is fluorescently labeled by the co-expression of gpD-mTurquoise 2 and wild type $\mathrm{gpD}$ (the capsid decoration protein) on the phage capsid, and the packaged phage DNA is fully methylated [28]. The host CRISPR/control strains (LZ1437/LZ1436) constitutively express a fluorescent SeqA fusion, SeqA-YFP, and the host DNA is not methylated owing to a dam mutation (methylation deficient) [22]. SeqA specifically binds to fully methylated and hemi-methylated DNA, so the fully methylated DNA of phage $\lambda_{\text {LZ760 }}$ forms an YFP focus once ejected 
A

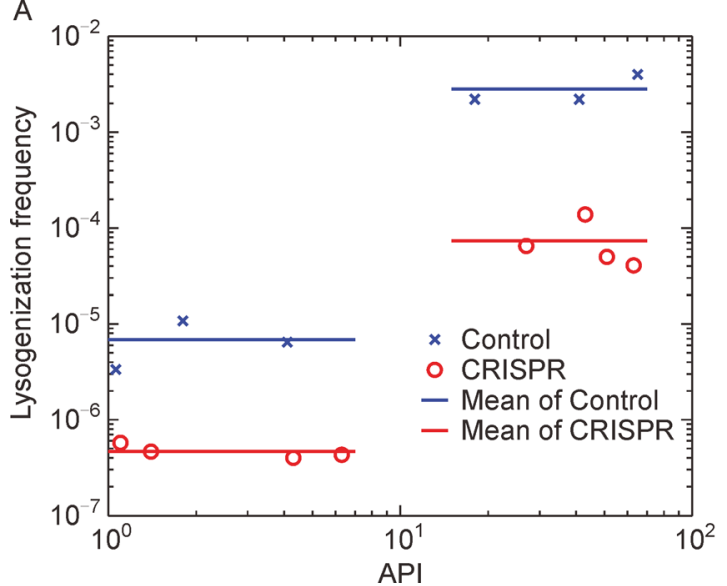

B

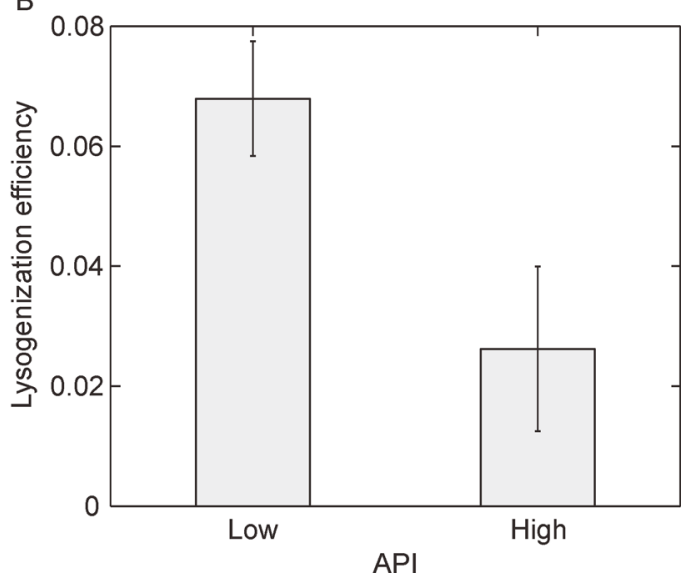

Figure 1. CRISPR system reduces lysogenization efficiency. (A) The lysogenization frequencies of CRISPR versus control system for our phage DNA reporter strain at a range of APIs (low API of $1-6$ and high API of 15-65). The control spacer: blue cross marker with blue lines as the mean for low and high APIs; the CRISPR spacer: red circle with red lines as the mean of the low and high APIs. (B) The lysogenization efficiency of the CRISPR system, defined as the ratio of average lysogenization frequency of the CRISPR system relative to that of the control system for low and high APIs. Error bar represents S.D.

into the cell. This reporter system allows the tracking of each initial phage DNA and its first, hemi-methylated, replicated copy. However, the replicated DNAs are not always necessarily visualized as two separate fluorescent spots since the replicated DNAs can stick together and spatially overlap, or stay not far away from each other, thus appearing as one spot during imaging under the microscope.

To determine how efficiently CRISPR functions, we compared the cell fates after phage infection in both control movies (i.e., cells containing three plasmids: pWUR397A (cas3), pWUR400 (casABCDE) and pWUR477 (control spacers)) and CRISPR movies (i.e., cells containing three plasmids: pWUR397A (cas3), pWUR400 (casABCDE) and pWUR478 ( $\lambda$ phage targeting spacers)). A successful phage infection is indicated by the appearance of a YFP focus during imaging. In the control movies (592 cells in 7 movies), 398 cells (67\%) start with one YFP focus staying with one focus throughout the movies (120 minutes) (classified as group 1), 186 cells (31\%) splitting into two foci (group 2 ), and 8 cells (2\%) splitting into 3 or more probably due to recombination (group 3) [23]. In the CRISPR movies (866 cells in 12 movies), there are 647 cells $(75 \%)$ in group 1, 206 cells (24\%) in group 2, and 13 cells (1\%) in group 3. For the simplicity of spot tracking, we only analyzed cells with only one YFP focus throughout the movies (cells in group 1). Two typical cell fates are observed: lytic $(86 \%, 341$ out of 398 cells in the control movies; $66 \%, 425$ out of 647 cells in the CRISPR movies) and CRISPR cells $(26 \%, 167$ out of 647 cells in the CRISPR movies). There are also rare cases of lysogeny or non-growing cells in both types of movies. The lytic cells show the accumulation of the fluorescent lytic reporter, gpD-mTurquoise 2 protein inside the cell. The CRISPR cells are defined as those that have phage DNA with much shorter SeqA-YFP spot lifetime and do not lyse, instead show normal cell growth. Figure 2 shows the snapshots of representative cells over time. At 0 min, phage DNA of a fluorescently labeled phage (appears as a red focus on the cell surface) has been ejected into the cell forming a YFP focus (appears as a green focus). Over time, the fluorescence intensity of the phage DNA (green or yellow focus, yellow color shown in the overlay images is due to the overlay of green and red fluorescence) decreases. DNA foci then disappear at $110 \mathrm{~min}$ (the lytic cell in the control movie), 120 min (the lytic cell in the CRISPR movie) and $45 \mathrm{~min}$ (the CRISPR cell in the CRISPR movie). The lytic cells eventually lyse, releasing fluorescent phage particles outside the cell while the CRISPR cell divides and grows normally like the uninfected cell. The time-lapse movies are shown in Supplementary Movies S1-S3. Five more examples of each fate are shown in Supplementary Movies S4-S6. As our movies only last 2 hours, some lytic cells do not lyse by the end of the movies, but the lytic reporter (gpD-mTurquoise2, appeared as red color) accumulates greatly indicating their lytic fate.

\section{Factors associated with effective CRISPR degradation}

To determine and quantify the degradation rate of DNA by CRISPR in each cell, we plot the spot intensity over time. The spot intensity in the cell population mostly maximizes within 5 minutes from the beginning of the 


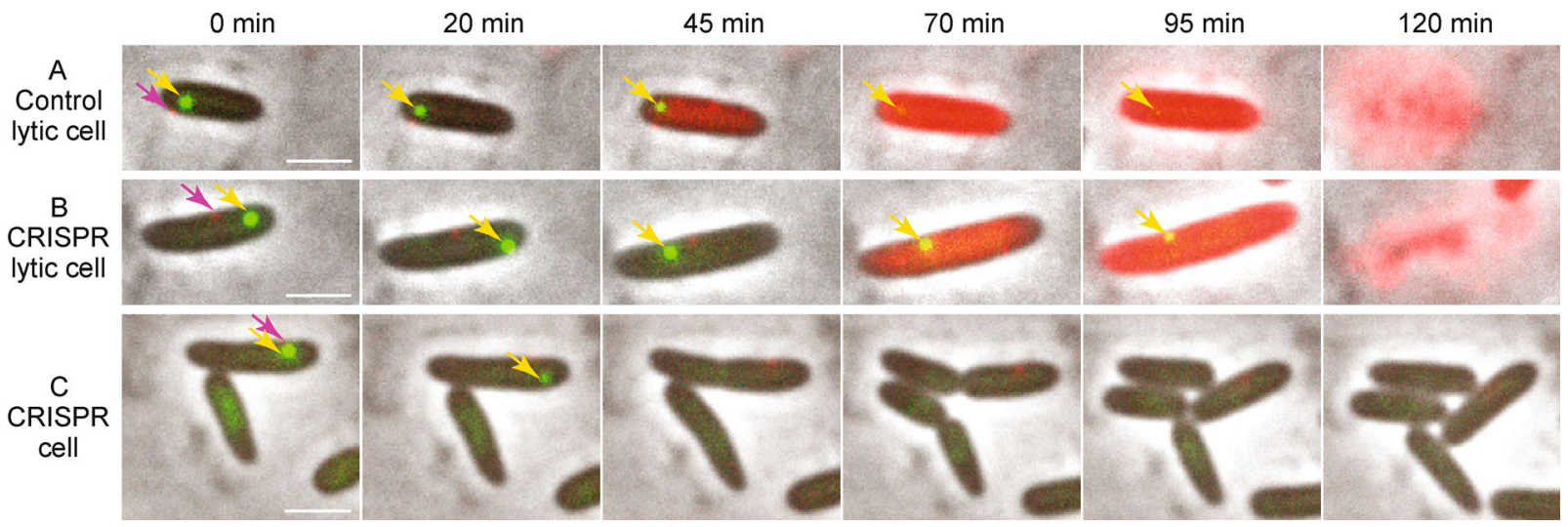

Figure 2. CRISPR system apparently degrades labeled phage DNA in single cells. The DNA of the fluorescently labeled infecting phage (red spot, pointed by purple arrows) is ejected into the host $E$. coli cell forming a fluorescent spot (green spot, pointed by yellow arrows) at $0 \mathrm{~min}$. The phage DNA (green spot) intensity decreases over time and finally disappears. (A) A lytic cell in a control movie. The green or yellow spot (yellow color is a result of overlay by green and red fluorescence) indicating the phage DNA disappears around $110 \mathrm{~min}$. At $120 \mathrm{~min}$, the cell lyses. (B) A lytic cell in a CRISPR movie. The green or yellow spot indicating the phage DNA disappears around 120 min or persists until cell lysis. At $120 \mathrm{~min}$, the cell lyses. (C) A CRISPR cell (top) and uninfected cell (bottom) in a CRISPR movie. The green spot indicating the phage DNA in the CRISPR cell disappears around 45 min, which is much earlier than that in the lytic cells in (A) and (B). At $70 \mathrm{~min}$, the cell divides, similar to that of the uninfected cell.

movie indicating that phage ejection is fairly synchronized among cells, shown in Supplementary Figure S1. The small variation in the timing of maximum intensity is likely due to the variability of DNA ejection time and the time delay from setting up our microscopy movies $[23,29]$. In addition, the average maximum DNA focus intensities in lytic cells are similar to those in CRISPR cells in CRISPR movies, therefore all cells have similar levels of SeqA-YFP binding prior to CRISPR functioning independent of cell fate (Supplementary Figure S2). To simplify the analysis and eliminate the variance of the maximum intensities among cells, we normalized spot intensities at each time point by the maximum intensity of each trace. We find that the spot intensity in CRISPR cells decreases much faster than that in lytic cells, shown in Figure $3 \mathrm{~A}$, which indicates that CRISPR is functioning to degrade labeled phage DNA. The DNA foci become undetectable by $43.9 \pm 1.5$ minutes to provide an estimated degradation rate (Figure 3B). Lytic cells also show fluorescence signal loss, but this is probably due to photobleaching, which provides a control for the effect of photobleaching under our experimental conditions. The spots in lytic cells usually disappear much later than those in CRISPR cells or the disappearance is not observed over the course of the movies (120 minutes) (Figure 3C). The spot disappearance in lytic cells is likely due to the phage DNA packaging, which would strip SeqA-YFP proteins off the phage DNA. As shown in Figure 3D, the spot disappearance time is correlated with cell lysis time with a correlation coefficient of 0.91 , p-value of 0.01 . When we compare the lytic cells in CRISPR movies with those in control movies, their fluorescence decreases similarly indicating that CRISPR spacers can fail in targeting the invading DNA, similarly to the control spacers (Supplementary Figure S3). Under our experimental conditions, the efficiency of CRISPR in targeting and degrading the phage DNA is around 26\%, 167 CRISPR-active cells out of 647 cells with one focus throughout the movies.

We next examined various factors associated with the time of CRISPR spot disappearance corresponding to the degradation of invading phage DNA. Considering the initial maximum spot intensity, due to time variation of taking movies, both between experiments and different stages within the same movie (up to a few minutes), the maximum intensity captured for each cell trace may correspond to a partially degraded phage DNA. If this were the case, the maximum intensity would be expected to have a positive correlation with spot disappearance time. Indeed, when we plot spot maximum intensity against disappearance time, we find the correlation coefficient to be 0.97 with p-value of 0.03 (Figure 4A). Next, we examined the effect of initial cell size, as cell size (or volume, or length as proxy) influences protein concentration, here the Cas proteins. Different concentrations of Cas proteins may degrade phage DNA at different rates. From our experiments, it seems the phage degradation does not change with cell size (correlation coefficient of -0.55 , with a large p-value of 0.45 ) as shown in Figure 4B. This indicates that the expressed Cas proteins are sufficiently concentrated to degrade phage DNA. Finally, we examined how phage DNA location inside the cell affects CRISPR action. Previous work has showed that phage lambda tends to adsorb to the cell pole and eject its DNA [30-32]. Although the exact mechan- 

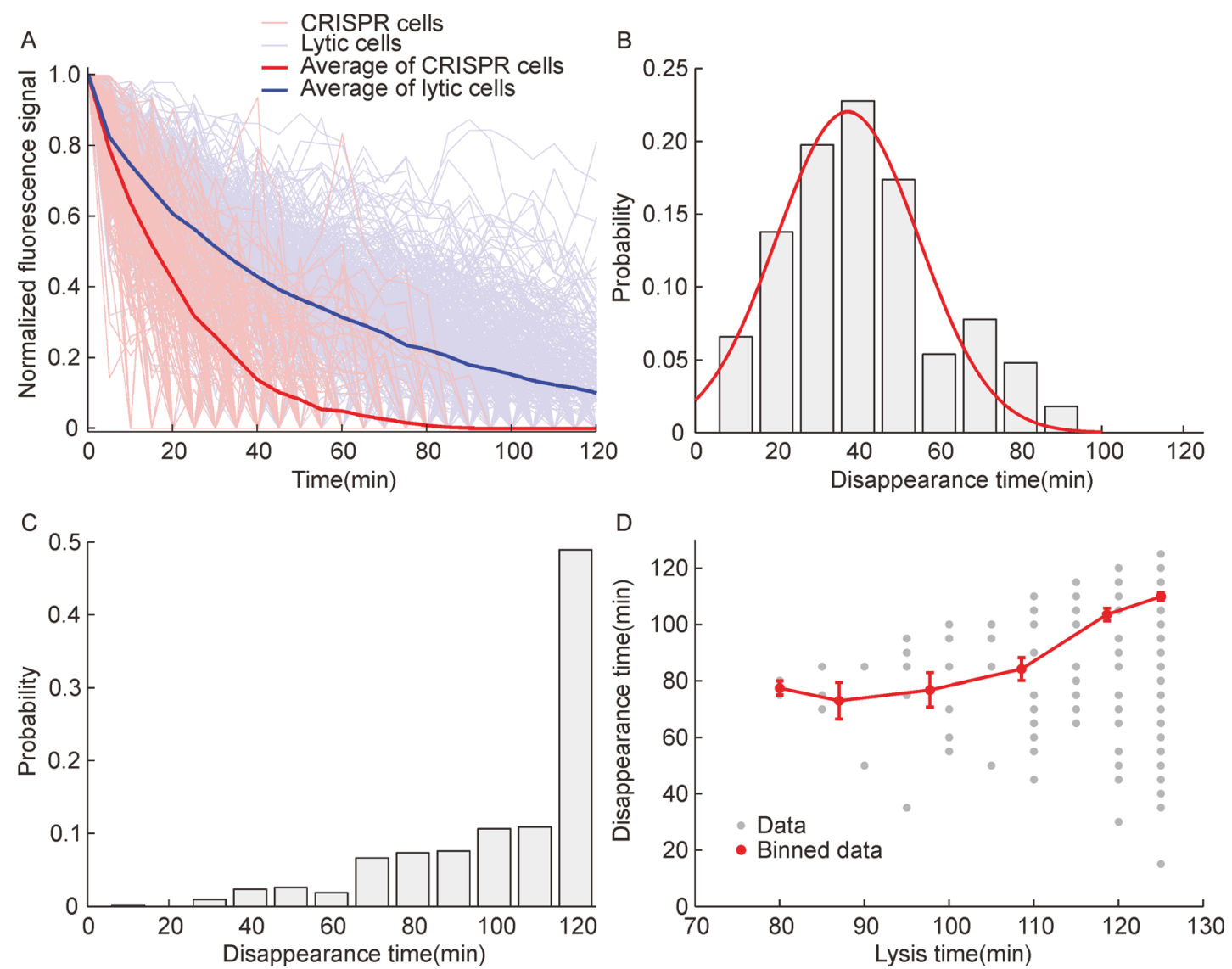

Figure 3. Phage DNA intensity decreases faster in CRISPR-active cells. (A) In the CRISPR movies, the phage DNA intensities of the CRISPR cells (red line, $N=167$ ) decrease much faster than those of the lytic cells (blue line, $N=423$ ) indicating CRISPR is actively functioning to degrade the invading phage DNA. The averages are shown as the thick lines. (B) The histogram of phage DNA spot disappearance time corresponding to the degradation time for CRISPR cells is well fitted to a Gaussian distribution (red line). The time to totally degrade phage DNA is around $43.9 \pm 1.5$ minutes. (C) The histogram of phage DNA spot disappearance time accounting for the photobleaching and/or phage DNA packaging into the phage head. Around $50 \%$ of the lytic cells still have the phage DNA spot at the end of the movies (120 min). (D) The phage DNA spot disappearance time is correlated with cell lysis time with a correlation coefficient of 0.91 , p-value of 0.01 . Error bar represents S.E.M.

ism underlying this polar preference has not been uncovered, cell pole is a special location where many proteins associated with the infection cycle, like ManYZ and FtsH accumulate [30]. We then ask whether the degradation of phage DNA inside the cell has any correlation with the initial phage DNA location. After the phage is ejected inside the cell, our recent work has shown that some phage DNAs move locally while others move over the whole cell [23]. When we correlate the phage DNA location with the efficiency of CRISPR degradation, we find that there appears to be no difference in CRISPR efficiency, whether the initial phage location is at polar/ mid-cell (future pole) or non-polar positions $(27 \% \pm 4 \%$ at pole versus $25 \% \pm 2 \%$ at non-pole, $p=0.02$ ) (Figure $4 \mathrm{C}$ ). Additionally, the timing of phage DNA degradation (spot disappearance time) is unaffected by phage DNA location $(42.1 \pm 2.4 \mathrm{~min}$ at pole/mid-cell and $44.7 \pm 1.8 \mathrm{~min}$ at non- pole, $\mathrm{p}=0.02)($ Figure $4 \mathrm{D})$. This indicates that the Cas proteins can effectively target invading DNA regardless of where the DNA is located.

\section{DISCUSSION}

In this study, we used our recently developed fluorescence technique to visualize phage DNA inside the cell upon infection by phage lambda to explore single-cell CRISPR function. With an artificial type I CRISPR system expressed in the host, the CRISPR system is able to degrade the invading phage DNA effectively with an efficiency of around $26 \%$ at the single-cell level. Consistent with the literature, the CRISPR system decreases the lysogenization frequency of the phage to be around $2 \%-7 \%$, which shows the effectiveness of CRISPR system degrading the invading phage DNA. To 

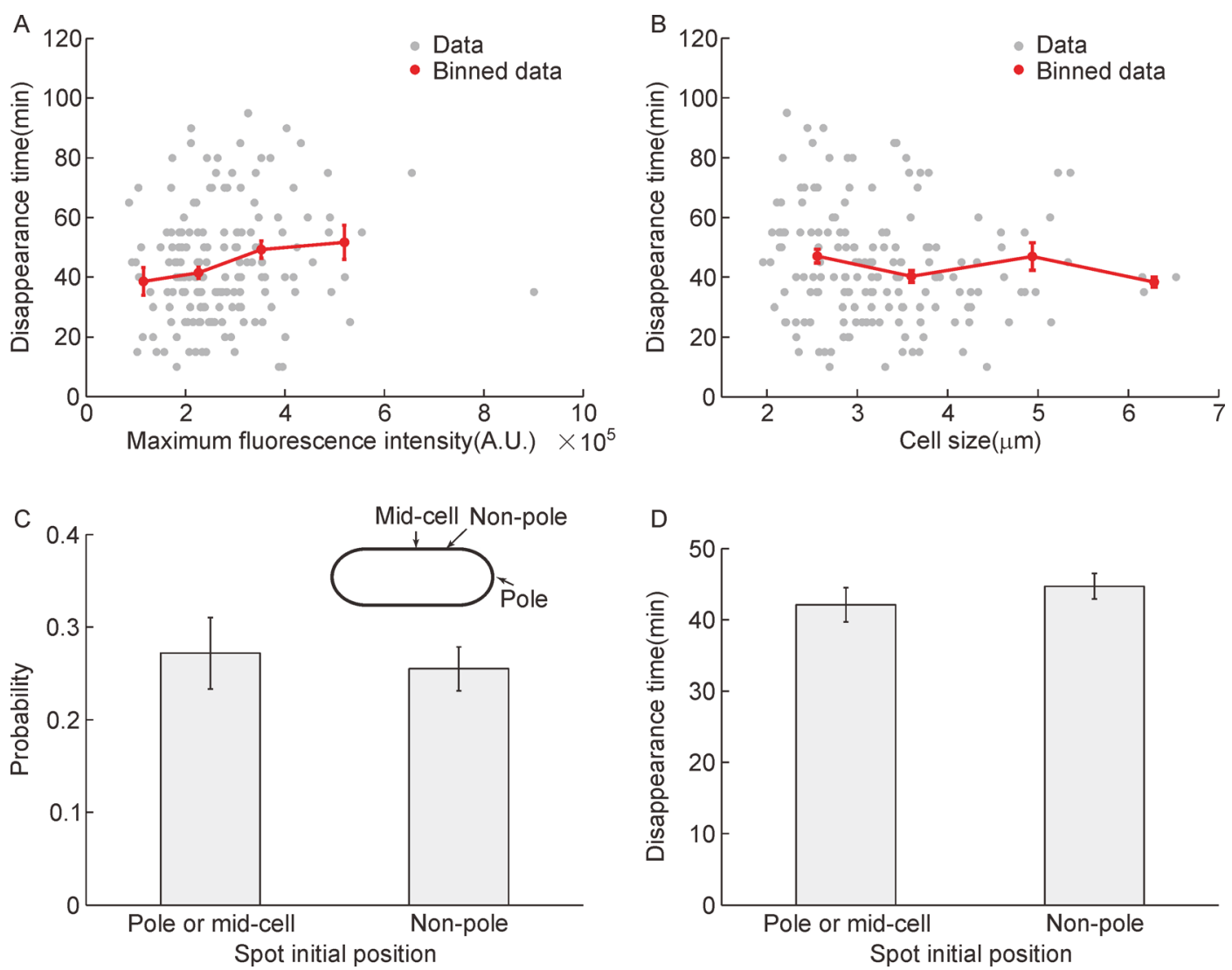

\begin{abstract}
Figure 4. Phage DNA degradation correlates with spot intensity, not with cell size or initial DNA location. (A) The complete phage DNA degradation or spot disappearance time positively correlates with the maximum intensity of the spot at the beginning of the movie with a correlation coefficient of 0.97 , p-value of 0.03 . The binned data were obtained with a bin interval of $1.5 \pm 10^{5} \mathrm{~A} . \mathrm{U}$. (B) The spot disappearance time does not change with the initial cell size with a correlation coefficient of $-0.55, p$-value of 0.45 . The binned data were obtained with a bin interval of $1 \mu \mathrm{m}$. (C) The efficiency of CRISPR is very similar for the initial invading phage DNA at polar/mid-cell $(0.27 \pm 0.04)$ or non-polar $(0.25 \pm 0.02)$ positions with a p-value of 0.02 . The diagram of the cell is shown on the top right. (D) The spot disappearance time does not seem to correlate with the initial phage DNA location showing similar disappearance time for polar/mid-cell $(42.1 \pm 2.4 \mathrm{~min})$ or non-polar $(44.7 \pm 1.8 \mathrm{~min}$ ) cell location with a p-value of 0.02. Error bar represents S.E.M.
\end{abstract}

our surprise, the fluorescent DNA foci in CRISPR cells do not divide into several smaller foci upon degradation, since the targeting plasmid pWUR478 contains four antilambda spacers with homology to lambda genes $J, O, R$, and $E$, which are fairly far apart from each other. If each spacer is targeted, the expected result would be that the phage genome is broken into multiple pieces, resulting in a few small fluorescent foci. However, our time-lapse movies show that the initial fluorescent DNA focus always stays as one before it finally disappears. Therefore, it is possible that only one anti-lambda spacer can target the phage DNA effectively one time with a synergistic effect of individual spacers and the Cas proteins degrade the DNA from that site thereafter, which may be due to the different sensitivities of the phage lambda DNA to the anti-lambda spacers [20]. But we cannot rule out the possibility that our SeqA-YFP system holds the cut DNA together somehow such that there is only one visible spot.
Under our experimental conditions under the fluorescence microscope, the lambda DNA takes about 44 minutes to be degraded (Figure $3 \mathrm{~A}$ and $3 \mathrm{~B}$ ). As a comparison, in in vitro experiments, a $5 \mathrm{~kb}$ negatively supercoiled DNA and linear DNA can be degraded within a few minutes ( 1 min and $5 \mathrm{~min})$ [11]. The much longer lambda DNA, $48.5 \mathrm{~kb}$ compared to this $5 \mathrm{~kb}$ DNA, almost 10 -fold longer, might be the major cause why it takes almost 10 -fold or more time to be digested. In addition, another contributor of different degrading time is the different experimental conditions of the in vitro experiments versus our in vivo experiments under the fluorescence microscope. The average fluorescence decrease in the lytic cells in the control and CRISPR movies (Supplementary Figure S4) is very similar, probably representing the photobleaching effect. The cell-to-cell variability of all the individual cells is probably due to the fluorescently labeled phage DNA 
moving in and out of focus, stochastic photobleaching of fluorescent molecules on the attached phage DNA and some unknown cell-to-cell variability. In addition to the stochastic photobleaching effect, the cell-to-cell variability of the CRISPR cells might also come from how easily the invading lambda DNA, probably the supercoiled form, is exposed for the Cas proteins to dock and degrade (Figure 3A).

From our single-cell analysis, the time to fully degrade the invading phage DNA is dependent on the initial fluorescent intensity of the phage DNA at the beginning of the movie. The lower maximum intensity of a spot in the cell might correspond to a partially degraded phage DNA due to the time to set up a movie, which is consistent with a fast initiation of CRISPR action. However, the phage DNA degradation does not depend on the initial cell size or phage DNA location. It is reasonable that Cas proteins are well mixed inside the cell and have high enough concentration to degrade the invading phage DNA with no preference of the location.

\section{MATERIALS AND METHODS}

\section{Bacterial strains, plasmids and phages}

The CRISPR/control strains (LZ1437/LZ1436) are BA16 (or MG1655 seqA-yfp dam ${ }^{-}$) [22] harboring three plasmids pWUR397A $\left(\operatorname{cas}_{3}, A m p^{R}\right)$ [21], pWUR400 (casABCDE, $\operatorname{Str}^{R}$ ) and pWUR478 $(\lambda$ phage targeting spacers, $\mathrm{Cm}^{R}$ )/pWUR477 (control spacers, $\mathrm{Cm}^{R}$ ) [20].

Fully methylated phage $\lambda_{\text {LZ760 }}$ was produced by inducing the lysogen MG1655( $\lambda$ D-mturqoise $2 \mathrm{cI}_{857}$ bor::Kan ${ }^{R}$ )[pBR322-pLate- $\left.D\right]$ harboring a Dam methylase overproducing plasmid pZA32-dam (with $1 \mathrm{mM}$ IPTG induction) [28]. The crude lysate was then purified from a standard $\mathrm{CsCl}$ purification procedure following the protocol described in [33].

All bacterial strains, plasmids and phages are listed in Table 1.

\section{Fluorescence microscopy and imaging}

An overnight culture of the host CRISPR/control strains LZ1437/LZ1436 was diluted $1: 100$ in M9 minimal medium $\left(11.3 \mathrm{~g} / \mathrm{L}\right.$ M9 salts, $1 \mathrm{mM} \mathrm{MgSO}_{4}, 0.5 \mu \mathrm{g} / \mathrm{mL}$ thiamine $\mathrm{HCl}, 0.1 \%$ casamino acids, $100 \mu \mathrm{M} \mathrm{CaCl} 2$ ) supplemented with $0.4 \%$ maltose (M9M) at $37^{\circ} \mathrm{C} .0 .2 \%$ L-Arabinose and $0.1 \mathrm{mM}$ IPTG were added into the culture to induce CRISPR plasmids for about 1.5 hours. When cells were grown to $\mathrm{OD}_{600} \approx 0.3$, and then concentrated and resuspended in ice-cold M9M with $0.2 \%$ L-Arabinose and $0.1 \mathrm{mM}$ IPTG to $\mathrm{OD}_{600} \approx 3$. $\lambda_{\text {LZ760 }}$ phages were added to reach a desired API $(10 \mu \mathrm{L}$ of cells $+10 \mu \mathrm{L}$ of phages), followed by incubation on ice for $30 \mathrm{~min}$ and an additional $5 \mathrm{~min}$ incubation at $35^{\circ} \mathrm{C}$ to trigger phage DNA ejection. The phage-cell mixture was diluted 1:10 in M9 with $0.2 \%$ L-Arabinose and $0.1 \mathrm{mM}$ IPTG, and $1 \mu \mathrm{L}$ of the diluted phage-cell mixture was placed on a thin $1.5 \%$ agarose slab of M9M with $0.2 \% \mathrm{~L}-$ Arabinose and $0.1 \mathrm{mM}$ IPTG ( $\sim \mathrm{mm}$ thick). After $1 \mathrm{~min}$, a coverslip (No.1.5, Fisher Scientific) was gently laid over the mixture and the sample was imaged under the fluorescence microscope at $30^{\circ} \mathrm{C}$ with a cage incubator (InVivo Scientific, St. Louis, MO). Microscopy was performed on an inverted epifluorescence microscope (Ti-E, Nikon, Tokyo, Japan) using a $100 \times$ objective (Plan Fluo, NA 1.40 , oil immersion) with a $2.5 \times \mathrm{TV}$ relay lens and standard filter sets. Images were acquired using a cooled EMCCD camera (iXon3 897, Andor, Belfast, United Kingdom). Acquisition was performed using Nikon Elements software.

To localize all phages surrounding the cells, a series of

Table 1. The bacteria, plasmids and phages used in this study.

\begin{tabular}{|c|c|c|}
\hline \multicolumn{3}{|c|}{ Bacterial strains, plasmids, and phages } \\
\hline Strain name & Relevant genotype & Source/Ref. \\
\hline \multicolumn{3}{|c|}{ Bacterial strains } \\
\hline BA16 & MG1655, dam ${ }^{-}$, seqA-yfp, $\mathrm{Cm}^{R}$ & {$[22]$} \\
\hline LZ1436 & BA16[pWUR397A, pWUR400, pWUR477], $\mathrm{Amp}^{R}, \mathrm{Str}^{R}, \mathrm{Cm}^{R}$ & This work \\
\hline LZ1437 & BA16[pWUR397A, pWUR400, pWUR478], $\mathrm{Amp}^{R}, \mathrm{Str}^{R}, \mathrm{Cm}^{R}$ & This work \\
\hline \multicolumn{3}{|l|}{ Phage strains } \\
\hline$\overline{\lambda_{\mathrm{LZ760}}}$ & Fully methylated, gpD-mosaic, $\lambda$ D-mTurquoise $2 I_{857}$ bor::Kan ${ }^{R}$ & {$[28]$} \\
\hline \multicolumn{3}{|l|}{ Plasmids } \\
\hline pWUR397A & cas 3 in $\mathrm{pRSF}-1 \mathrm{~b}$ & {$[21]$} \\
\hline pWUR400 & $\operatorname{cas} A$-cas $B$-cas $C$-cas $D$-cas $E$ in $\mathrm{pCDF}-1 \mathrm{~b}$ & {$[20]$} \\
\hline pWUR477 & Non-targeting CRISPR/spacers from E. coli $\mathrm{K} 12$ with no homology to phage lambda in pACYCDuet-1 & {$[20]$} \\
\hline pWUR478 & Template CRISPR/template strand of lambda genes $J, O, R$ and $E$ in pACYCDuet-1 & {$[20]$} \\
\hline
\end{tabular}


$5 \mathrm{z}$-axis (vertical) images at a spacing of $400 \mathrm{~nm}$ were taken through the mTurquoise2/CFP channel using $200 \mathrm{~ms}$ exposure. To obtain more data in each timelapse movie, cells were imaged at multiple stage positions (typically 16) in each experiment. During the time-lapse movie, the sample was imaged in phase contrast $(100 \mathrm{~ms}$ exposure, for cell recognition), YFP (200 ms exposure, for phage DNA detection inside the cell, with a series of 5 z-axis images at a spacing of $400 \mathrm{~nm}$ in order to localize phage DNA foci), mTurquoise2/CFP (200 ms exposure, for phage lytic reporter) channels. The time-lapse movies were taken at a time interval of $5 \mathrm{~min}$, until the cell fate was observable (2 hours).

\section{Data analysis}

All data analysis was performed in Matlab (The MathWorks, Natick, MA). Images were processed using MicrobeTracker [34]. Briefly, cells were first outlined by the phase-contrast channel using MicrobeTracker, after which spots were recognized first automatically using SpotFinderZ, then manually corrected using SpotFinderM [34]. Cell lineage tracking and fluorescent intensity were processed and calculated using our home-made Matlab script.

\section{Bulk lysogenization assay}

To measure the lysogenization frequency, we followed the protocol as described in [32]. Briefly, $2 \mathrm{~mL}$ of the host CRISPR/control strains LZ1437/LZ1436 was grown in LBMM $\left(\mathrm{LB}+0.2 \%\right.$ maltose $\left.+10 \mathrm{mM} \mathrm{MgSO}_{4}\right)$ with appropriate antibiotics for overnight and subsequently diluted 1:100 into $12 \mathrm{~mL}$ of LBMM and grown at $37^{\circ} \mathrm{C}$. $0.2 \% \mathrm{~L}$-Arabinose and $0.1 \mathrm{mM}$ IPTG were added into the culture to induce CRISPR plasmids for about 1.5 hours. When grown to $\mathrm{OD}_{600} \approx 0.4$, cells were centrifuged $\left(1000 \times g\right.$ for $10 \mathrm{~min}$ at $\left.4^{\circ} \mathrm{C}\right)$, concentrated $10 \times$ and resuspended to $\mathrm{OD}_{600} \approx 4$ in pre-chilled LBMM with $0.2 \%$ L-Arabinose and $0.1 \mathrm{mM}$ IPTG. $20 \mu \mathrm{L}$ of the resuspended cells were then infected with $20 \mu \mathrm{L}$ of phages at different concentrations by incubation for $30 \mathrm{~min}$ on ice. The samples were then transferred to $35^{\circ} \mathrm{C}$ water bath for $5 \mathrm{~min}$ to allow for phage DNA ejection, followed by 100 -fold dilution into pre-warmed LBGM $\left(\mathrm{LB}+0.2 \%\right.$ glucose $\left.+10 \mathrm{mM} \mathrm{MgSO}_{4}\right)$ with $0.2 \%$ L-Arabinose and $0.1 \mathrm{mM}$ IPTG and incubation with shaking at $265 \mathrm{rpm}$ at $30^{\circ} \mathrm{C}$ for $45 \mathrm{~min}$. The samples were then properly diluted and plated on LB + Kan plates to allow around 100 colonies to grow.

\section{SUPPLEMENTARY MATERIALS}

The supplementary materials can be found online with this article at DOI 10.1007/s40484-017-0099-0.

\section{ACKNOWLEDGEMENTS}

We are grateful to Rodem Edgar for providing the CRISPR plasmids. We would like to thank all members of the Zeng laboratory for help with the experiments and data analysis. Work in the Zeng laboratory was supported by the National Institutes of Health (R01GM107597). The funder had no role in study design, data collection and interpretation, or the decision to submit the work for publication.

\section{COMPLIANCE AND ETHICS GUIDELINES}

Jingwen Guan, Xu Shi, Roberto Burgos, and Lanying Zeng declare that they have no conflict of interest.

This article does not contain any studies with human or animal subjects performed by any of the authors.

\section{REFERENCES}

1. Mohanraju, P., Makarova, K. S., Zetsche, B., Zhang, F., Koonin, E. V. and van der Oost, J. (2016) Diverse evolutionary roots and mechanistic variations of the CRISPR-Cas systems. Science, 353, aad5147

2. Deveau, H., Garneau, J. E. and Moineau, S. (2010) CRISPR/Cas system and its role in phage-bacteria interactions. Annu. Rev. Microbiol., 64, 475-493

3. van Erp, P. B., Jackson, R. N., Carter, J., Golden, S. M., Bailey, S and Wiedenheft, B. (2015) Mechanism of CRISPR-RNA guided recognition of DNA targets in Escherichia coli. Nucleic Acids Res., 43, 8381-8391

4. Bhaya, D., Davison, M. and Barrangou, R. (2011) CRISPR-Cas systems in bacteria and archaea: versatile small RNAs for adaptive defense and regulation. Annu. Rev. Genet., 45, 273-297

5. Sternberg, S. H., Richter, H., Charpentier, E. and Qimron, U. (2016) Adaptation in CRISPR-Cas systems. Mol. Cell, 61, 797-808

6. van der Oost, J., Westra, E. R., Jackson, R. N. and Wiedenheft, B. (2014) Unravelling the structural and mechanistic basis of CRISPR-Cas systems. Nat. Rev. Microbiol., 12, 479-492

7. Huo, Y., Nam, K. H., Ding, F., Lee, H., Wu, L., Xiao, Y., Farchione, M. D. Jr, Zhou, S., Rajashankar, K., Kurinov, I., et al. (2014) Structures of CRISPR Cas3 offer mechanistic insights into Cascade-activated DNA unwinding and degradation. Nat. Struct. Mol. Biol., 21, 771777

8. Hatoum-Aslan, A., Maniv, I. and Marraffini, L. A. (2011) Mature clustered, regularly interspaced, short palindromic repeats RNA (crRNA) length is measured by a ruler mechanism anchored at the precursor processing site. Proc. Natl. Acad. Sci. USA, 108, 21218 21222

9. Sinkunas, T., Gasiunas, G., Fremaux, C., Barrangou, R., Horvath, P and Siksnys, V. (2011) Cas3 is a single-stranded DNA nuclease and ATP-dependent helicase in the CRISPR/Cas immune system. EMBO J., 30, 1335-1342

10. Barrangou, R. (2015) Diversity of CRISPR-Cas immune systems and molecular machines. Genome Biol., 16, 247

11. Mulepati, S. and Bailey, S. (2013) In vitro reconstitution of an Escherichia coli RNA-guided immune system reveals unidirectional, ATP-dependent degradation of DNA target. J. Biol. Chem., 288, 22184-22192

12. Amitai, G. and Sorek, R. (2016) CRISPR-Cas adaptation: insights into the mechanism of action. Nat. Rev. Microbiol., 14, 67-76 
13. Künne, T., Kieper, S. N., Bannenberg, J. W., Vogel, A. I., Miellet, W. R., Klein, M., Depken, M., Suarez-Diez, M. and Brouns, S. J. (2016) Cas3-derived target DNA degradation fragments fuel primed CRISPR adaptation. Mol. Cell, 63, 852-864

14. McGinn, J. and Marraffini, L. A. (2016) CRISPR-Cas systems optimize their immune response by specifying the site of spacer integration. Mol. Cell, 64, 616-623

15. Jackson, R. N., Golden, S. M., van Erp, P. B., Carter, J., Westra, E. R., Brouns, S. J., van der Oost, J., Terwilliger, T. C., Read, R. J. and Wiedenheft, B. (2014) Crystal structure of the CRISPR RNA-guided surveillance complex from Escherichia coli. Science, 345, 1473-1479

16. Hochstrasser, M. L., Taylor, D. W., Bhat, P., Guegler, C. K., Sternberg, S. H., Nogales, E.and Doudna, J. A. (2014) CasA mediates Cas3catalyzed target degradation during CRISPR RNA-guided interference. Proc. Natl. Acad. Sci. USA, 111, 6618-6623

17. Westra, E. R., van Erp, P. B., Kunne, T., Wong, S. P., Staals, R. H., Seegers, C. L., Bollen, S., Jore, M. M., Semenova, E., Severinov, K., et al. (2012) CRISPR immunity relies on the consecutive binding and degradation of negatively supercoiled invader DNA by Cascade and Cas3. Mol. Cell, 46, 595-605

18. Mulepati, S., Heroux, A. and Bailey, S. (2014) Crystal structure of a CRISPR RNA-guided surveillance complex bound to a ssDNA target. Science, 345, 1479-1484

19. Redding, S., Sternberg, S. H., Marshall, M., Gibb, B., Bhat, P., Guegler, C. K., Wiedenheft, B., Doudna, J. A. and Greene, E. C. (2015) Surveillance and processing of foreign DNA by the Escherichia coli CRISPR-Cas system. Cell, 163, 854-865

20. Brouns, S. J., Jore, M. M., Lundgren, M., Westra, E. R., Slijkhuis, R. J., Snijders, A. P., Dickman, M. J., Makarova, K. S., Koonin, E. V. and van der Oost, J. (2008) Small CRISPR RNAs guide antiviral defense in prokaryotes. Science, 321, 960-964

21. Edgar, R. and Qimron, U. (2010) The Escherichia coli CRISPR system protects from lambda lysogenization, lysogens, and prophage induction. J. Bacteriol., 192, 6291-6294

22. Babic, A., Lindner, A. B., Vulic, M., Stewart, E. J. and Radman, M. (2008) Direct visualization of horizontal gene transfer. Science, 319, $1533-1536$
23. Shao, Q., Hawkins, A. and Zeng, L. (2015) Phage DNA dynamics in cells with different fates. Biophys. J., 108, 2048-2060

24. Pul, U., Wurm, R., Arslan, Z., Geissen, R., Hofmann, N. and Wagner, R. (2010) Identification and characterization of E. coli CRISPR-cas promoters and their silencing by H-NS. Mol. Microbiol., 75, 14951512

25. Lu, M., Campbell, J. L., Boye, E. and Kleckner, N. (1994) SeqA: a negative modulator of replication initiation in E. coli. Cell, 77, 413-426

26. Slater, S., Wold, S., Lu, M., Boye, E., Skarstad, K. and Kleckner, N. (1995) E. coli SeqA protein binds oriC in two different methylmodulated reactions appropriate to its roles in DNA replication initiation and origin sequestration. Cell, 82, 927-936

27. Pougach, K., Semenova, E., Bogdanova, E., Datsenko, K. A., Djordjevic, M., Wanner, B. L. and Severinov, K. (2010) Transcription, processing and function of CRISPR cassettes in Escherichia coli. Mol. Microbiol., 77, 1367-1379

28. Shao, Q., Trinh, J. T., McIntosh, C. S., Christenson, B., Balazsi, G. and Zeng, L. (2017) Lysis-lysogeny coexistence: prophage integration during lytic development. MicrobiologyOpen, 6

29. Van Valen, D., Wu, D., Chen, Y. J., Tuson, H., Wiggins, P. and Phillips, R. (2012) A single-molecule Hershey-Chase experiment. Curr. Biol., 22, 1339-1343

30. Edgar, R., Rokney, A., Feeney, M., Semsey, S., Kessel, M., Goldberg, M. B., Adhya, S. and Oppenheim, A. B. (2008) Bacteriophage infection is targeted to cellular poles. Mol. Microbiol., 68, 1107-1116

31. Rothenberg, E., Sepulveda, L. A., Skinner, S. O., Zeng, L., Selvin, P. R. and Golding, I. (2011) Single-virus tracking reveals a spatial receptor-dependent search mechanism. Biophys. J., 100, 2875-2882

32. Zeng, L., Skinner, S. O., Zong, C., Sippy, J., Feiss, M. and Golding, I. (2010) Decision making at a subcellular level determines the outcome of bacteriophage infection. Cell, 141, 682-691

33. Zeng, L. and Golding, I. (2011) Following cell-fate in E. coli after infection by phage lambda. J. Vis. Exp., 56, e3363,

34. Sliusarenko, O., Heinritz, J., Emonet, T. and Jacobs-Wagner, C. (2011) High-throughput, subpixel precision analysis of bacterial morphogenesis and intracellular spatio-temporal dynamics. Mol. Microbiol., 80, 612-627 